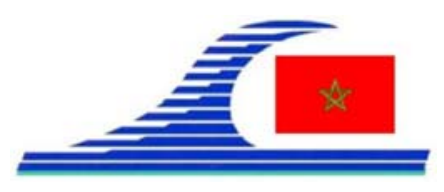

Conférence Méditerranéenne Côtière et Maritime

EDITION 2, TANGER, MAROC (2011)

Coastal and Maritime Mediterranean Conference

Disponible en ligne - http://www.paralia.fr - Available online

\title{
Caractérisation numérique et expérimentale des interactions entre deux hydroliennes
}

\author{
Paul MYCEK ${ }^{1,2}$, Benoît GAURIER ${ }^{2}$, Grégory GERMAIN ${ }^{2}$, \\ Corentin LOTHODÉ ${ }^{1,2}$, Grégory PINON ${ }^{1}$, Élie RIVOALEN ${ }^{1,3}$
}

1. Université du Havre, UFR Sciences et Techniques, FRE 3102 CNRS - LOMC, 53 rue de Prony, BP 540, 76058 Le Havre, France.

gregory.pinon@univ-lehavre.fr

2. IFREMER, Centre Manche Mer du Nord, Service Hydrodynamique et Océanométéo, 150 quai Gambetta, BP 699, 62321 Boulogne-sur-Mer, France.

\{paul.mycek, benoit.gaurier,gregory.germain\}@ifremer.fr

3. INSA de Rouen, EA 3828 - LMR,

Avenue de l'Université, BP 08, 76801 Saint-Étienne-du-Rouvray, France.

elie.rivoalen@insa-rouen.fr

\section{Résumé :}

L'implantation et le développement de fermes d'hydroliennes sont soumis à la compréhension des effets d'interaction entre de telles machines. En nous inspirant de suggestions a priori sur la disposition de fermes de convertisseurs d'énergie marine, nous nous proposons de mettre en évidence les interactions entre deux hydroliennes.

Des essais expérimentaux ont été réalisés dans le canal à houle et courant de l'IFREMER à Boulogne-sur-Mer, sur des maquettes d'hydrolienne. Notre étude se concentre sur des configurations où la seconde hydrolienne est placée en alignement dans le sillage de la première, à différentes distances l'une de l'autre. Les effets d'interaction sont mis en évidence à la fois en termes de performances et de caractérisation du sillage grâce à une comparaison avec des résultats obtenus sur des configurations avec une seule hydrolienne. L'étude montre que le comportement de la seconde hydrolienne est largement influencé par la présence de la première.

Nous présentons également des résultats numériques sur une seule hydrolienne obtenus à partir de notre code de simulation tridimensionnel développé au Laboratoire Ondes et Milieux Complexes. La validation de ces résultats par rapport aux essais expérimentaux correspondants nous permet d'envisager une très prochaine prise en compte de plusieurs machines et ainsi la modélisation de fermes à géométrie plus complexe.

\section{Mots-clés :}

Énergies marines - Hydroliennes à axe horizontal - Fermes d'hydroliennes - Essais expérimentaux - Laser Doppler Velocimetry - Simulation numérique - Méthode vortex 
La connaissance de la Mer :

un vecteur du développement durable en Méditerranée

\section{Introduction}

Le comportement d'une hydrolienne soumis à différentes conditions d'entrée est désormais assez bien documenté grâce notamment à des études expérimentales (BATTEN et al., 2008 ; MAGANGA et al., 2010) ou numériques (BALTAZAR \& FALCÃO DE CAMPOS, 2008). En revanche, la question des interactions entre hydroliennes est assez peu traitée dans la littérature. Si l'on peut trouver quelques remarques a priori sur l'implantation de fermes de convertisseurs d'énergie marine (MYERS et al., 2010), aucune étude dans des conditions réelles de fonctionnement n'a été menée sur des hydroliennes à axe horizontale à notre connaissance. Nous nous proposons de poser les premières pierres de ce travail en nous intéressant aux interactions entre deux hydroliennes placées l'une derrière l'autre.

Dans une première partie, nous exposons des résultats numériques et expérimentaux sur une seule hydrolienne qui nous serviront dans une deuxième partie à mettre en évidence l'existence d'effets d'interactions entre deux hydroliennes alignées.

\section{Configuration avec une seule hydrolienne}

Le comportement d'une hydrolienne est influencé par différents paramètres, parmi lesquels :

- La vitesse amont, uniforme dans la présente étude, notée $U_{\infty}$;

- Le Tip Speed Ratio (TSR), définie comme le ratio entre la vitesse en bout de pale et la vitesse de l'écoulement amont $U_{\infty}$ :

$$
T S R=\frac{\Omega R}{U_{\infty}}
$$

où $R=D / 2$ est le rayon du rotor et $\Omega$ est sa vitesse de rotation;

- Le taux de turbulence ambiant (TI), défini par

$$
T I=100 \times \frac{\sqrt{u^{\prime 2}+v^{\prime 2}+w^{\prime 2}}}{\sqrt{\bar{u}^{2}+\bar{v}^{2}+\bar{w}^{2}}}
$$

où $u, v, w$ sont les trois composantes du vecteur vitesse, $\bar{q}$ est la moyenne et $q^{\prime}$ l'écart-type de la quantité $q$.

Des essais ont été réalisés dans le bassin d'essais de l'IFREMER de Boulogne-sur-Mer sur une maquette à l'échelle $1 / 30$ d'un prototype d'hydrolienne. La description de la maquette est donnée par le tableau 1.

Tableau 1. Caractéristiques de la maquette d'hydrolienne.

\begin{tabular}{ll|ll}
\hline Caractéristique & Description & Caractéristique & Description \\
\hline Profil des pales & NACA63418 & TSR étudiés & {$[0 ; 10]$} \\
Diamètre du rotor $(D)$ & $700 \mathrm{~mm}$ & Sens de rotation & Antihoraire \\
Diamètre de la nacelle & $92 \mathrm{~mm}$ & Reynolds $\left(\mathrm{Re}_{\infty}\right)$ & $\approx 280000$ \\
Longueur de la nacelle & $720 \mathrm{~mm}$ & & \\
\hline
\end{tabular}




\subsection{Le sillage}

Grâce à un système de LDV (Laser Doppler Velocimetry), la vitesse moyenne est évaluée en différents points en aval de l'hydrolienne. Avec une grille suffisamment fine de points, on peut alors tracer des cartes de sillages. La figure 1 montre deux cartes de vitesse axiale moyenne en aval de l'hydrolienne; pour la première (figure 1(a)), l'hydrolienne était plongée dans un écoulement avec un taux de turbulence ambiant de $5 \%$, tandis que dans la seconde (figure 1(b)), celui-ci était bien plus élevé (25\%). On peut déduire de ces cartes que la turbulence ambiante influence considérablement le sillage de l'hydrolienne. En effet, pour un taux de turbulence élevé (25\%), l'écoulement tend à retrouver son uniformité et sa vitesse initiale à partir de 7 diamètres derrière l'hydrolienne; en revanche, avec un taux de turbulence de $5 \%$, le sillage reste très prononcé même après 10 diamètres derrière l'hydrolienne.

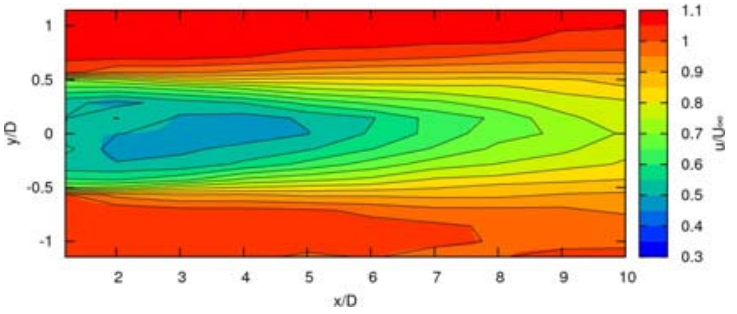

(a) Carte de vitesse axiale $(T I=5 \%)$

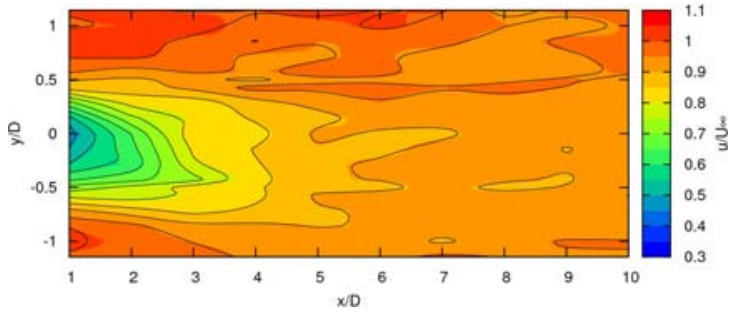

(b) Carte de vitesse axiale $(T I=25 \%)$

Figure 1. Cartes de vitesse axiales pour différents TI, avec TSR=3,67.

À partir de ces profils on peut estimer la valeur moyenne de la vitesse axiale $\hat{u}$ à une certaine distance $x$ derrière l'hydrolienne en intégrant la vitesse axiale à cette distance sur l'aire d'influence de rayon $R^{*}$ de l'hydrolienne :

$\hat{u}(x)=\frac{1}{R^{*}} \int_{-R^{*}}^{+R^{*}}|y| u(x, y) d y$

Ici, $R^{*}$ est pris légèrement plus grand que $R$ de manière à ce que tout le déficit de vitesse induit par la présence de l'hydrolienne soit bien pris en compte. Le déficit moyen en $x$ est alors naturellement défini par

$\gamma(x)=100 \times\left(1-\hat{u}(x) / U_{\infty}\right)$

Cette quantité nous permet de mieux comparer nos résultats numériques et expérimentaux. Le code utilisé est un code développé par notre équipe au Laboratoire Ondes et Milieux Complexes de l'Université du Havre. Il est basé sur une méthode vortex et présenté en détail dans (PINON et al., 2005 ; MAGANGA et al., 2010 ; PINON et al., soumis). La figure 2 montre une carte de vitesse axiale obtenue numériquement pour une configuration correspondant à un TI de $0 \%$ (figure $2(\mathrm{a})$ ) et la comparaison du déficit de vitesse derrière l'hydrolienne avec les résultats expérimentaux (figure 2(b)). Les résultats numériques sont en bon accord avec 
La connaissance de la Mer :

un vecteur du développement durable en Méditerranée

l'expérience. Les différences observées proviennent du fait qu'un TI ambiant ne peut pas encore être pris en compte dans le code numérique et que le modèle de turbulence implémenté dans le code n'est pas suffisamment sophistiqué.

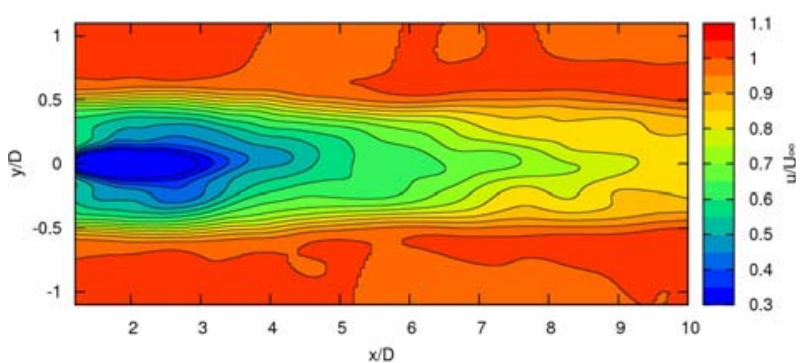

(a) Carte de vitesse axiale $(\mathrm{TI}=0 \%)$

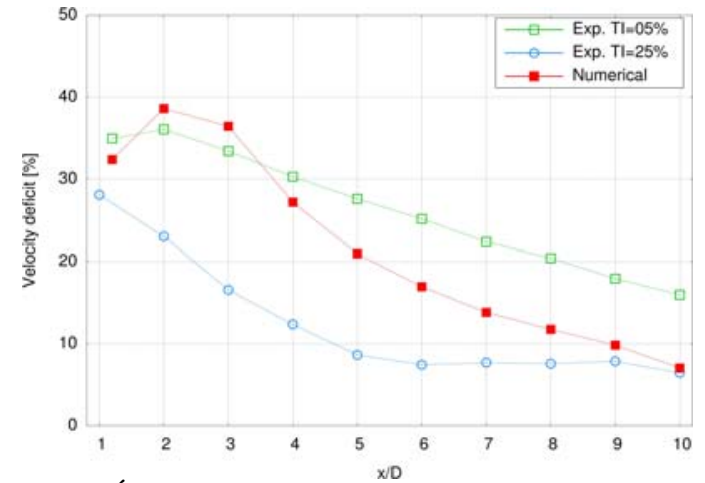

(b) Évolution du déficit de vitesse axiale en fonction de la distance à l'hydrolienne

Figure 2. Caractérisation numérique du sillage, avec $T S R=3,67$.

\subsection{Les performances}

Les performances d'une hydrolienne peuvent être évaluées par le biais de son coefficient de puissance défini par

$$
C_{P}=\frac{M_{x} \Omega}{\frac{1}{2} \rho \pi R^{2} U_{\infty}^{3}}
$$

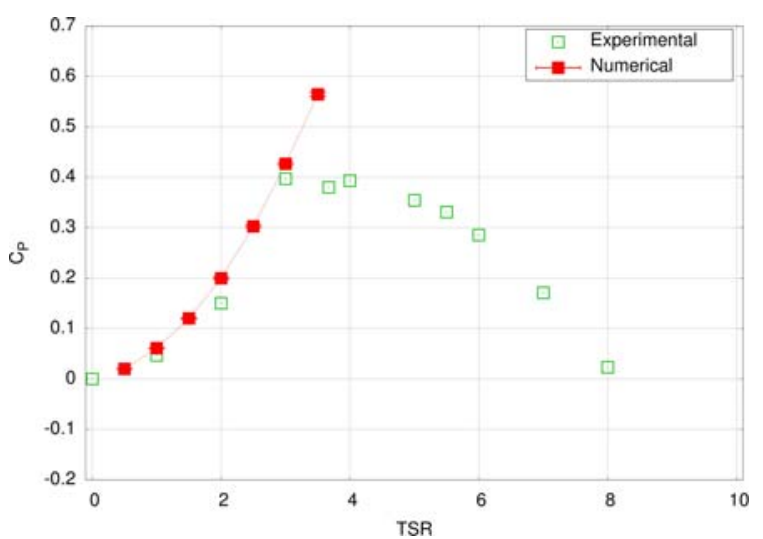

Figure 3. Évolution $d u C_{P}$ en fonction $d u$ TSR.

La figure 3 montre l'évolution du coefficient de puissance de l'hydrolienne en fonction de son TSR. Le point de fonctionnement est atteint entre $\mathrm{TSR}=3$ et $\mathrm{TSR}=4$, avec près de $40 \%$ d'énergie récupérée $\left(C_{P}=0,4\right)$. L'évolution numérique est en adéquation avec l'évolution expérimentale jusqu'à $\mathrm{TSR}=3$. Le fait que le $C_{P}$ numérique continue de croître est dû à notre modèle d'émission particulaire qui ne prend pas actuellement en compte le décollement de couche limite sur les pales de l'hydrolienne. 


\section{Interactions entre deux hydroliennes}

Notre étude se concentre sur les interactions entre deux hydroliennes placées l'une dans le sillage de l'autre. Les axes des rotors sont alignés avec le courant, comme le montre la figure 4, qui décrit le dispositif expérimental.

La figure 5(a) montre la carte de vitesse axiale derrière l'hydrolienne aval placée à $4 D$ derrière la première. Le TI mesuré en amont des deux hydroliennes est de 5\%. Par ailleurs, le TI mesuré à une distance de $4 D$ derrière une seule hydrolienne est de $25 \%$. On pourrait alors s'attendre à obtenir derrière la deuxième hydrolienne un sillage similaire à celui obtenu pour une seule hydrolienne plongée dans un TI de $25 \%$, figure 1(b). La différence considérable que l'on peut observer entre ces deux cartes (5(a) et 1(b)) met en évidence les effets d'interaction entre les deux hydroliennes.

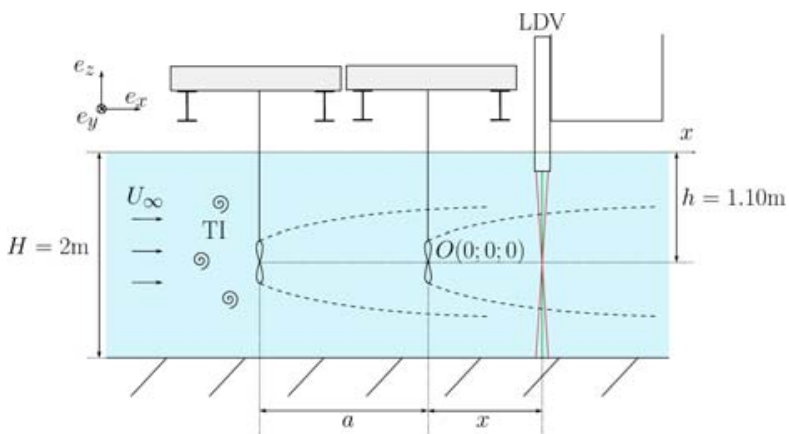

(a) Vue de côté

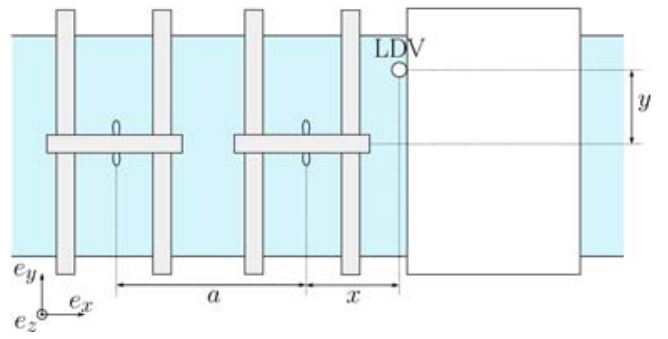

(b) Vue de dessus

Figure 4. Vues schématiques du dispositif expérimental.

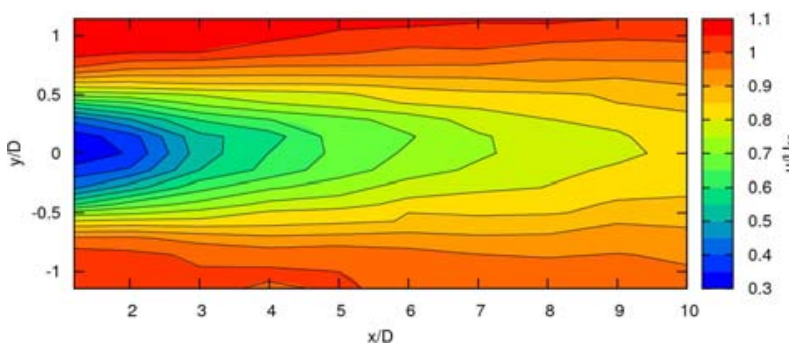

(a) Carte de vitesse axiale derrière l'hydrolienne aval (TI=5\%). Les deux hydroliennes sont espacées d'une distance de $a=4 D$.

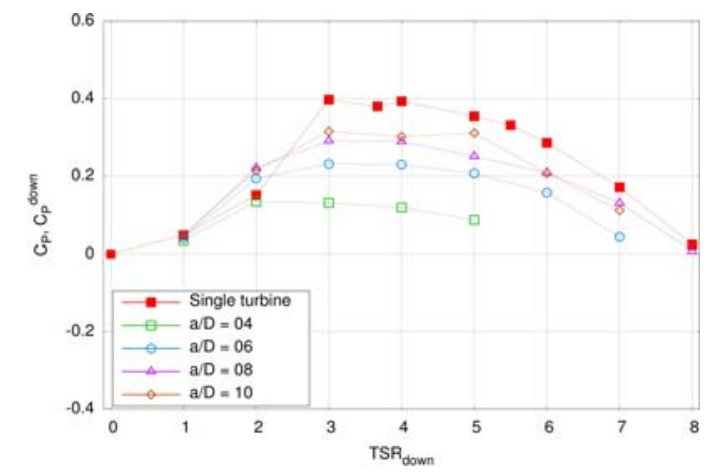

(b) Évolution du $C_{P}^{\text {down }}$ de l'hydrolienne aval en fonction de son $T S R_{\text {down }}$.

Figure 5. Mise en évidence des interactions entre deux hydroliennes

En ce qui concerne les performances, la figure 5(b) montre pour différentes valeurs de $a / D$ le $C_{P}$ de l'hydrolienne aval en fonction de son $T S R_{\text {down }}$. Le $T S R_{\text {down }}$ est calculé grâce à la formule (1) c'est-à-dire toujours par rapport à la vitesse de courant $U_{\infty}$ en amont des deux hydroliennes. La comparaison avec le $C_{P}$ d'une seule hydrolienne montre que l'influence de l'hydrolienne amont sur l'hydrolienne aval diminue avec 
La connaissance de la Mer :

un vecteur du développement durable en Méditerranée

l'augmentation de la distance $a / D$. À une distance $a / D=10$, la seconde hydrolienne retrouve quasiment le même comportement qu'une hydrolienne seule.

\section{Conclusion et perspectives}

Cette étude a permis de mettre en évidence l'existence d'effets d'interaction entre deux hydroliennes alignées dans un courant uniforme. Les résultats sur les performances montrent en outre qu'un compromis est nécessaire entre la performance individuelle de chaque machine et la distance inter-hydroliennes.

Nos prochains travaux vont principalement se concentrer sur l'expansion et l'amélioration du code de calcul de manière à disposer d'un modèle de turbulence plus sophistiqué et à pouvoir prendre en compte plusieurs hydroliennes.

De nouveaux essais en bassin sont également prévus, notamment des études de sillage derrière deux hydroliennes avec un TI amont de $25 \%$.

\section{Remerciements}

Les auteurs souhaitent remercier la Région Haute-Normandie pour le soutien financier accordé aux co-financements de thèse et le CRIHAN pour la mise à disposition des moyens informatiques pour les calculs numériques. Nous tenons également à remercier Thomas Bacchetti et Jean-Valéry Facq pour leur aide dans le présent projet. Le code de simulation numérique a été initialement développé pour des applications aéronautiques dans le cadre de travaux avec l'entreprise Aircelle et la Région Haute-Normandie.

\section{Références bibliographiques}

BALTAZAR J., FALCÃO DE CAMPOS J. A. C. (2008). Hydrodynamic analysis of a horizontal axis marine current turbine with a boundary element method. 27th OMAE Conference, Estoril (Portugal), pp 883-893.

BATTEN W., BAHAJ A., MOLLAND A., CHAPLIN J. (2008), The prediction of the hydrodynamic performance of marine current turbines. Renewable Energy, vol. 33, $\mathrm{n}^{\circ}$ 5, pp 1085-1096.

MAGANGA F., GERMAIN G., KING J., PINON G., RIVOALEN E. (2010). Experimental characterisation of flow effects on marine current turbine behaviour and on its wake properties. IET Renewable Power Generation, vol. 4, n 6, pp 498-509.

MYERS L., BAHAJ A., RETZLER C., RICCI P., DHEDIN J.-F. (2010). Inter-device spacing issues within wave and tidal energy converter arrays. ${ }^{\text {rd }}$ ICOE, Bilbao.

PINON G., BRATEC H., HUBERSON S., PIGNOT G., RIVOALEN E. (2005). Vortex method for simulation of a $3 D$ round jet in a cross-stream. Journal of Turbulence, vol. $6, \mathrm{n}^{\circ} 18$, pp 1-25.

PINON G., MYCEK P., GERMAIN G., RIVOALEN R. (soumis). Numerical Simulation of the Wake of Marine Current Turbines with a Particle Method. Soumis à Renewable Energy. 